\title{
Alternating Product Ciphers: A Case for Provable Security Comparisons (extended abstract ${ }^{\star}$ )
}

\author{
John O. Pliam^ \\ Institute for Data Intensive Engineering and Science (IDIES), Johns Hopkins \\ University, 3400 N. Charles St., Baltimore, MD 21218, USA john.pliam@jhu.edu
}

\begin{abstract}
We formally study iterated block ciphers that alternate between two sequences of independent and identically distributed (i.i.d.) rounds. It is demonstrated that, in some cases the effect of alternating increases security, while in other cases the effect may strictly decrease security relative to the corresponding product of one of its component sequences. As this would appear to contradict conventional wisdom based on the ideal cipher approximation, we introduce new machinery for provable security comparisons. The comparisons made here simultaneously establish a coherent ordering of security metrics ranging from key-recovery cost to computational indistinguishability.
\end{abstract}

Keywords. block ciphers, product ciphers, multiple encryption, majorization.

\section{Introduction}

\subsection{Overview.}

For many decades, various issues related to product ciphers have been raised and addressed. A large part of Shannon's seminal work [16] is devoted to both theoretical and practical aspects of products, and his invocation of the pastry dough mixing analogy [16, p. 712] captures a very intuitive idea that by alternating between two weakly mixing operations, we should eventually achieve strong mixing. Even today, many modern block cipher designs retain an element of this structure (see, e.g. 8]).

To model such mixing, we formalize the notion of an alternating product cipher as an interleaving product of independent ciphers as depicted in Fig. 3. We then ask: How well does the mixing work, and how might it fail? Various outcomes seem possible. We present a threefold alternating product with good security expansion. However, a related construction demonstrates somewhat surprisingly, an alternating product which is strictly less secure than the two-term

* to appear in Indocrypt 2013.

** The author's position is supported in part by the U.S. National Science Foundation

(NFS) Data-Scope grant CISE/ACI 1040114. 
product of one of the component sequences by itself. On its face, this would appear to contradict an emerging conventional wisdom about multiple encryption based on the ideal cipher approximation, "that double encryption improves the security only marginally [...] triple encryption is significantly more secure than single and double encryption" [6]. The situation demands that we explore the problem of provable security comparisons. We find that certain security orderings transcend the (somewhat artificial) boundary between classical and modern cryptography.

We conclude that alternating product ciphers are, at a fundamental level, different from two-term products. Ascertaining their security is more nuanced and they provide evidence of further limits on the applicability of the ideal cipher approximation (see also [1]).

\subsection{Motivation.}

We are initially motivated by how we might generalize the question, "is a cipher a group?", in the case of alternating products. Roughly, an encryption function $E: K \times M \rightarrow M$ is said to have the group property [16, p.673], if for each key pair $\left(k_{1}, k_{2}\right)$, there is another key $k \in K$ such that $E\left(k_{2}, E\left(k_{1}, p\right)\right)=E(k, p)$ for each plaintext $p \in M$. Equivalently, the product of the cipher with itself produces no new permutations.

The group property obviously affords the cryptanalyst considerable advantage, if only because it reduces the cost of brute-force search against the product. Understandably, the question was raised as a possible weakness to multiple encryption schemes of DES [7]. These concerns were dismissed with increasing strength as researchers showed that DES was not likely to be a group [7, that it is not a group [3] and that it generates a large group [18].

Questions about whether a cipher is a group or whether multiple encryption improves security are really questions about ordering. That is to say, rather than quantifying specific models of attack against fixed encryption systems, we seek to establish the correct ordering between constructs of interest. In the case of alternating products, we find the comparison between $X Y Z$ and $X Z$ to be the most intriguing since our intuition suggests that inserting statistically independent $Y$ in between $X$ and $Z$ should improve security. Thus in comparing the two products of Fig. 1, we find that the order itself depends on the internal structure of the constituents.

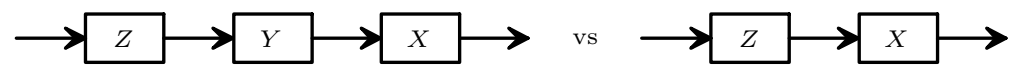

Fig. 1. Motivating comparison between alternating product $X Y Z$ and $X Z$. 


\subsection{Toward Coherent Security Ordering.}

Motivated by the above, we start by quantifying how the permutation count of an alternating product can grow or shrink. Numerically comparing these counts offers one possible ordering, since integers are totally ordered (every pair is comparable). But we argue that by relaxing this notion and considering partial/pre orders on ciphers, we pave the way to stronger and more broadly applicable security comparisons. There is a lucrative trade-off here: if we give up comparing every pair of ciphers, we are left with a more meaningful ordering of the remainder.

One powerful order (known to not be total) is majorization, and a great many interesting real-valued security metrics are known to respect majorization. These are called Schur-convex (concave if they reverse it). This covers the case of zero data complexity in the far left of Fig. 22 if a majorization relationship between two ciphers can be established, then the ciphers are also ordered by the real values of any Schur-convex(concave) function.

Better still, the comparisons of Fig. 1 in this paper possess additional structure, facilitating a coherent ordering of security metrics in arbitrary data complexity $q$. Specifically, we show in Sect. 3, that nonadaptive chosen-plaintext attack (ncpa) advantage [11/17] as well as conditional guesswork 13] are such metrics. This is depicted in the two rightmost diagrams in Fig. 2
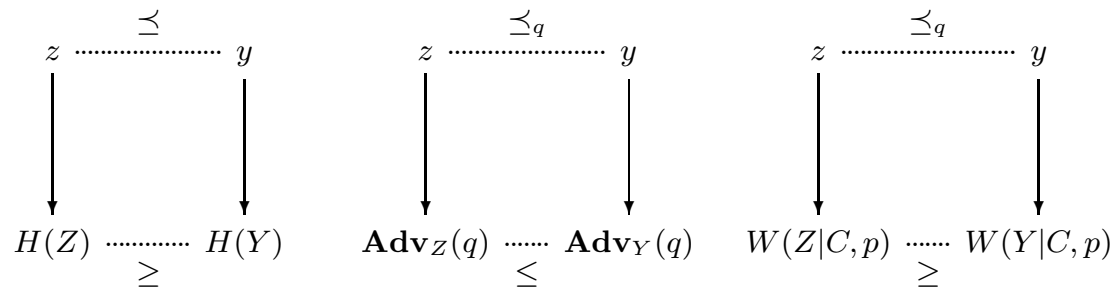

Fig. 2. We establish a coherent ordering of the ciphers in Fig. 1 by showing the consistency of a broad range of security metrics, crossing the divide between informationtheoretic and modern cryptography.

\section{Preliminaries}

\subsection{Prerequisites.}

We've tried to make this paper readable by nonspecialists conversant in contemporary cryptography. But in order to follow the proofs, we assume additional familiarity with the basics of permutation groups, probability theory and representation theory, referring the reader to [15], [5]. We also exploit aspects of the theory of majorization, treated very well in [9. Since that is critical here, we provide a brief summary in 3.1 


\subsection{Shannon's Model.}

We formalize Shannon's model [16 by representing an encryption system as a permutation-valued random variable. Precisely, given message space $\mathcal{M}$, let $G$ be some subgroup of the full symmetric group $\mathfrak{S}_{\mathcal{M}}$ of all permutations on $\mathcal{M}$. An encryption system on $\mathcal{M}$, or $G$-cipher for short, is a $G$-valued random variable $X$. As a notational convention, for a $G$-cipher $X$ (always uppercase), we shall denote the probability distribution from which it is drawn by lowercase function $x: G \rightarrow \mathbb{R}$ and write $X \sim x$.

Shannon observed that the set of encryption systems is endowed with the structure of an unital associative algebrd 11 whose sum and product correspond to parallel and series composition, respectively. The composition in series of independent $G$-ciphers $X, Y$ gives the notion of a product cipher $Z=X Y$, which survives to this day. It is a standard observation that the probability distribution of the product $z(g)=\operatorname{Pr}[X Y=g]$, is given by the convolution $z=x * y$ :

$$
z(g)=\sum_{h \in G} x\left(g h^{-1}\right) y(h) .
$$

\section{Models For Security Comparison}

After a brief review of the theory of majorization, we explore ways in which claims of security ordering may be rigorously established as in Fig. 2.

\subsection{Majorization and Schur-Convexity.}

Given vectors $x, y \in \mathbb{R}_{+}^{n}$ we say $x$ is majorized by $y$ and write $x \preceq y$, if their $l_{1}$ norms agree and for each $1 \leq k \leq n$,

$$
\sum_{i=1}^{k} x_{[i]} \leq \sum_{i=1}^{k} y_{[i]},
$$

with the values rearranged by $x_{[1]} \geq x_{[2]} \geq \cdots \geq x_{[n]}$ and similarly for $y$. The vector $x_{\downarrow}$ is the decreasing rearrangement of $x$ (so $\left.x_{[i]}=\left(x_{\downarrow}\right)_{i}\right)$. Majorization is a preorder relation, so not all vectors are comparable in this way. We have, from the Hardy-Littlewood-Pólya theorem, that $x \preceq y$ is equivalent to the existence of a doubly-stochastic matrix $D$ such that $x=D y$. Furthermore, by the Birkhoffvon Neumann theorem, such a matrix is a convex sum of permutations, so $x \preceq y$ means:

$$
x=\sum_{\pi \in \mathfrak{S}_{n}} p_{\pi} \pi \cdot y .
$$

For our purposes, the vectors will usually be probability distributions, each with $l_{1}$ norm of 1 . It is readily verified that the uniform distribution $u=(1 / n, \ldots, 1 / n)$

\footnotetext{
${ }^{1}$ These days, it would be identified as the group algebra $\mathbb{R} G$.
} 
has $u \preceq x$ for all probability vectors $x$. If $x \preceq y$ and $y \preceq x$, then $x$ is a permutation of $y$. If $x \preceq y$ but $x$ is not a permutation of $y$, we'll write $x \prec y$.

Certain useful real-valued functions respect or reverse majorization. So if $\phi$ : $\mathbb{R}_{+}^{n} \rightarrow \mathbb{R}$ has $\phi(x) \leq \phi(y)(\phi(x) \geq \phi(y))$ whenever $x \preceq y$, we call $\phi$ Schur-convex (concave). If a Schur-convex (concave) function additionally satisfies $\phi(x)<\phi(y)$ $(\phi(x)>\phi(y))$ when $x \prec y$, we call $\phi$ strictly Schur-convex (concave).

Examples and applications abound throughout science and engineering (see e.g. 4 for an interesting information-theoretic treatment). In particular, Shannon entropy, Rényi entropy and guesswork [10] are strictly Schur-concave. Furthermore marginal guesswork [12] and Bonneau's $\alpha$-guesswork 2] are Schurconcave. For further details, see [9, pp. 562-564] and [14, Appx.]. As remarked, majorization treats the case of zero data complexity, which is sometimes useful by itself.

\subsection{Nontrivial Data Complexity.}

For an adversary with access to $q$ plaintext-ciphertext pairs or equivalently $q$ queries to a chosen-plaintext oracle, we can often identify a vector mapping $\sigma: V \rightarrow \hat{V}$ and a Schur-convex function $\phi_{q}$ on $\hat{V}$ measuring in some way the cipher's resistance to attack. If $z \preceq y$ in $V$ has additional structure so that $\hat{z} \preceq \hat{y}$ in $\hat{V}$, we write $z \preceq_{\phi_{q} \circ \sigma} y$ or just $z \preceq_{q} y$ when clear from context. This situation affords meaningful security comparisons for arbitrary data complexity. A proof of the following is sketched in the appendix and proved in the full version [14.

Theorem 1 Given data complexity limit $q$ and $G$-ciphers $X \sim x, Y \sim y$ and $Z \sim z$ with $Z=X Y$, we have (for appropriate choices of $\sigma$ )

1. $z \preceq_{q} y$ for conditional guesswork: $W(E \mid C, p), p \in \mathcal{M}^{(q)}$ is Schur-concave as a function of $\hat{e}$,

2. $z \preceq_{q} y$ for distinguishing advantage: $\mathbf{A d v}_{E}^{\text {ncpa }}(q)$ is Schur-convex as a function of $\hat{e}$,

Here $E \sim e$ is a generic argument.

The relationship $z \preceq_{q} y$ can arise in many ways, but for our purposes, we'll use the fact that $Z=X Y$.

\section{Alternating Product Ciphers}

\subsection{The Formal Definition.}

We may now give a formal definition of an alternating product followed by an example.

Definition 1 An alternating product is the product of independent $G$-ciphers alternating between two sequences of i.i.d. G-ciphers. 
Example 1 Let $\left\{X_{i}\right\}_{i=1}^{r+1}$ be i.i.d. G-ciphers and let $\left\{Y_{i}\right\}_{i=1}^{r}$ be distinct i.i.d. $G$-ciphers. Then $E=X_{r+1} Y_{r} X_{r} \cdots Y_{1} X_{1}$ is an alternating product of $X_{i}$ and $Y_{i}$. Notice that Def. 1 permits either an even or an odd number of components in the product. We can imagine $E$ as alternating between the "factors" of two products $X=X_{r+1} \cdots X_{1}$ and $Y=Y_{r} \cdots Y_{1}$ as depicted in Fig. 圆 below.

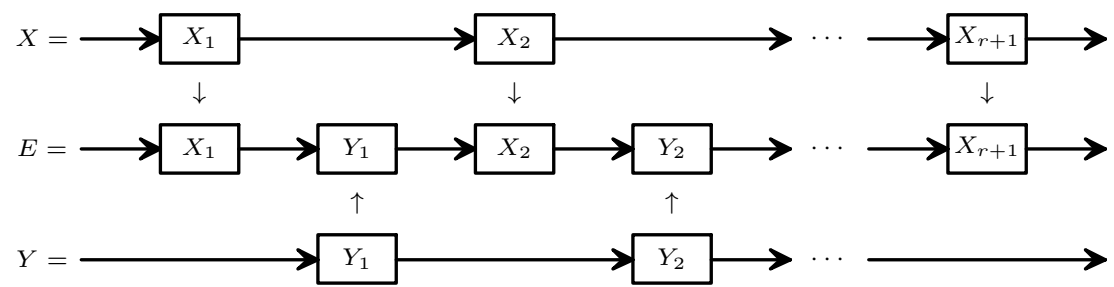

Fig. 3. An alternating product cipher seen as an interleaving of the terms of two iterated ciphers.

\subsection{Threefold Mixing Convolutions and Double Cosets.}

While most of this paper is devoted to alternating products, we treat a slightly more general case in this section to explicate a key observation, namely how mixing in typical iterated block ciphers is related to expansion along double cosets when randomness enters via a subgroup operation (like the XOR-ing of round subkeys).

Consider a threefold product $T=X Y Z$ with $Z$ confined to subgroup $K \leq G$, $X$ confined to subgroup $H \leq G$, and $Y$ deterministically taking single value $\pi \in$ $G$. To understand how the convolution $t=x * y * z$ decomposes, it's instructive to employ an action of $g \in G$ on functions $\phi: G \rightarrow \mathbb{R}$ taking $\phi \mapsto \phi \circ g^{-1}$, in other words $(g \cdot \phi)(f)=\phi\left(g^{-1} f\right)$. With this in mind, we have the following useful lemma.

Lemma 1 If the support of $\phi$ is confined to a left coset $k H$, then the support of $g \cdot \phi$ is confined to $g k H$.

Proof: Assume $\operatorname{supp}(\phi) \subseteq k H$, and observe that $f \in \operatorname{supp}(g \cdot \phi) \Longrightarrow(g \cdot \phi)(f) \neq$ $0 \Longrightarrow \phi\left(g^{-1} f\right) \neq 0 \Longrightarrow g^{-1} f \in \operatorname{supp}(\phi) \Longrightarrow g^{-1} f \in k H \Longrightarrow f \in g k H$. $\operatorname{Thus} \operatorname{supp}(g \cdot \phi) \subseteq g k H$.

It should be intuitively clear that $T$ is spread out over the double coset $H \pi K$, but the following stronger result details some of the mechanics of the mixing, facilitating deeper security comparisons. 
Theorem 2 The cipher $T$ has $\operatorname{supp}(t) \subseteq H \pi K$ and its distribution is a convex direct sum

$$
t=\bigoplus_{i=1}^{m} \alpha_{i} z_{i}
$$

of $m=\left[H: H \cap^{\pi} K\right]$ distinct probability vectors. Furthermore, each $z_{i}$ is majorized by $z$.

Proof: The three terms in $T=X Y Z$ are given by probability distributions $x(g), y(g)$ and $z(g)$ with $\operatorname{supp}(x) \subseteq H, \operatorname{supp}(y)=\{\pi\}$ and $\operatorname{supp}(z) \subseteq K$. First note that by the associativity of product ciphers, we may write $T=X(Y Z)$, and the inner convolution $z^{\prime}=y * z$ yields,

$$
z^{\prime}(g)=\sum_{h \in G} y(h) z\left(h^{-1} g\right)=z\left(\pi^{-1} g\right)=\pi \cdot z(g),
$$

which by Lem. 1, precisely describes a function confined to $\pi K$.

Now the outer convolution $t=x * z^{\prime}$ yields

$$
t(g)=\sum_{h \in G} x(h) z^{\prime}\left(h^{-1} g\right)=\sum_{h \in H} x(h) z^{\prime}\left(h^{-1} g\right)=\sum_{h \in H} x(h) h \cdot z^{\prime}(g) .
$$

Recognizing that $z^{\prime}$ is confined to $\pi K$, it is natural according to Lem. 1 to collect terms for which $h \cdot z^{\prime}$ is confined to the same left coset of $K$. Indeed, recall that the left action of $H$ on left cosets $G / K$ (the supports of the various $\left.h \cdot z^{\prime}\right)$ is equivalent to the double coset action $H \backslash G / K$. We may decompose the orbit $H \pi K=\bigcup_{i=1}^{m} \lambda_{i} K$, where the orbit size $m=\left[H: H \cap{ }^{\pi} K\right]$ is given by the orbit-stabilizer theorem with

$$
S \triangleq \operatorname{Stab}_{H \backslash(G / K)}(\pi K)=H \cap{ }^{\pi} K .
$$

Furthermore, we recognize $\left\{h \in H \mid h(\pi K)=\lambda_{i} K\right\}=h_{i} S$, for some left transversal $\left\{h_{i}\right\}$ of $S$ in $H$. This gives us a recipe for collecting terms,

$$
\begin{aligned}
t=\sum_{h \in H} x(h) h \cdot z^{\prime}=\sum_{i=1}^{m} \sum_{h \in h_{i} S} x(h) h \cdot z^{\prime} & =\sum_{i=1}^{m} x\left(h_{i} S\right) \underbrace{\left(\frac{1}{x\left(h_{i} S\right)} \sum_{h \in h_{i} S} x(h) h \cdot z^{\prime}\right)}_{\triangleq z_{i}} \\
& =\sum_{i=1}^{m} x\left(h_{i} S\right) z_{i},
\end{aligned}
$$

where, by construction, each $z_{i}$ is confined to left $\operatorname{coset} \lambda_{i} K$, so the sum is a direct sum. By the Hardy-Littlewood-Pólya and Birkhoff-von Neumann theorems,

$$
z_{i}=\frac{1}{x\left(h_{i} S\right)} \sum_{h \in h_{i} S} x(h) h \cdot z^{\prime}
$$

is a convex sum of permuted copies of $z^{\prime}$, assuring majorization $z_{i} \preceq z^{\prime} \preceq z$. Finally, taking $\alpha_{i}=x\left(h_{i} S\right)$ yields $\sum_{i} \alpha_{i}=1$ and the theorem is proved.

Uniform distributions simplify the matter. 
Corollary 1 When both $X$ and $Z$ are uniformly distributed, $T$ is uniformly distributed on $H \pi K$.

Proof: The uniformity of $Z$ implies $z \preceq z_{i} \preceq z$, so each $z_{i}$ is uniform on $\lambda_{i} K$. The uniformity of $X$ implies

$$
t=\sum_{i=1}^{m} x\left(h_{i} S\right) z_{i}=\frac{|S|}{|H|} \sum_{i=1}^{m} z_{i}=\frac{\left|H \cap{ }^{\pi} K\right|}{|H|} \sum_{i=1}^{m} z_{i}=\frac{1}{m} \sum_{i=1}^{m} z_{i},
$$

which precisely describes a function uniform on $H \pi K$.

The following is immediate.

Corollary 2 If in addition to the conditions of the previous corollary, $|H \pi K|>$ $|K|$ then $t \prec z$.

REMARK. For noncommutative $G$, the additional condition in Coro. 2 is actually the typical case, even when $H=K$. For the remainder of this paper we assume $H \neq{ }^{\pi} H$ so that $\left[H: H \cap{ }^{\pi} H\right]>1$ and thus $|H \pi H|>|H|$. This is always true in a simple group since $H={ }^{\pi} H$ means $H$ is normal.

\section{Applications}

\subsection{An Expanding Alternating Product.}

If, in Coro. 22 we further impose $H=K$, we obtain an alternating product cipher in the sense of Def. 1 .

Proposition 1 The alternating product cipher $T=X Y Z$ is more secure than $D=X Z$ when $X$ and $Z$ are uniform $H$-ciphers and $Y$ is deterministic on $\{\pi\}$, in the sense that:

a). The mixing of permutations in $T$ produces dramatically more than $D$.

b). $t \prec d$, so by any strictly Schur-convave security metric, $T$ is more secure than $D$.

c). $t \preceq_{q} d$, so by the security metrics of Thm. 1, $T$ is no less secure than D.

Proof: For (a) and (b), we need only apply Coro. 1 and Coro. 2. For (c), observe that since $D=X Z=Z, T=X Y Z=(X Y) D$, we have the necessary product relation for Thm. 1 .

\subsection{A Collapsing Alternating Product.}

Now let $H$ be a subgroup of $G$ and let $\pi \in G$ fall strictly outside $H$ (so $H \neq \pi H$ ). Consider three independent $G$-ciphers $X, Y, Z$, where both $X$ and $Z$ are uniformly distributed on the left coset $\pi H$ and $Y$ takes the value $\pi^{-1}$ deterministically. We seek to compare the products $T=X Y Z$ vs $D=X Z$. Note that since $X$ and $Z$ are i.i.d., and $Y$ is independent of these, $T$ is also an alternating product. We have the following. 
Proposition 2 The product cipher $D$ is more secure than the alternating product $T$ in that:

a). The mixing of permutations in $D$ produces dramatically more than $T$.

b). $d \prec t$, so by any strictly Schur-convex security metric, $D$ is more secure than $T$.

c). $d \preceq_{q} t$, so by the security metrics of Thm. 目, D is no less secure than $T$.

Proof: Without loss of generality, we may drop the trailing $\pi$, which poses no cryptanalytic barrier. We compare instead the products $T^{\prime}=X^{\prime} Y Z$ and $D^{\prime}=$ $X^{\prime} Z$, with $X^{\prime}$ uniform on $H$. Writing $T^{\prime}=X^{\prime}(Y Z)$ the inner convolution $v=y * z$ trivially reduces to uniform on $H$. In this way, $T^{\prime}=X^{\prime} V$ is the double encryption of Prop. 1. On the other hand, $D^{\prime}$ is uniform on $H \pi H$ since it is of the form of the triple product of Prop. 1 The desired result follows at once from this reversal of roles of the double and triple product from Prop. 1 .

\subsection{A Collapsing General Alternating Product.}

Consider again the general alternating product cipher of Fig. 3 and Ex. 1, only now with each $X_{i}$ uniform on $\pi H$ and each $Y_{i}$ deterministically taking $\pi^{-1}$. We seek to compare $E=X_{r+1} Y_{r} X_{r} \cdots Y_{1} X_{1}$ with $X=X_{r+1} \cdots X_{1}$. Again because products are associative, we may write $E=X_{r+1}\left(\left(Y_{r} X_{r}\right) \cdots\left(Y_{1} X_{1}\right)\right)$, and each of the inner convolutions $e_{i}=y_{i} * x_{i}$ trivially collapses to uniform on $H$. Further the sequence of convolutions $v=e_{r} * \cdots * e_{1}$ remains uniform on $H$ and the final $x_{r+1} * v$ is uniform on $\pi H$. On the other hand $x_{2} * x_{1}$ has support on translate of double coset $H \pi H$ and continued left convolution can only make this count go up. Clearly then, we have.

Proposition $3 X$ is more secure than $E$.

\subsection{A Resource-Bounded Example of Extreme Expansion.}

It may seem from our treatment in the above examples that the ciphers here are purely information theoretic, applying only to infeasible and hypothetical ciphers. In this section, we present a positive example of a computationally efficient alternating product cipher $T=X Y Z$ which has nearly optimal expansion of permutations along a huge double coset, yet where the $D=X Y$ is trivially distinguishable from any idealized cipher.

To facilitate such a comparison, we exploit special properties of a polynomialtime cipher which achieves every permutation (given enough key construction data or equivalently a private random function oracle).

This construction from [13] called a universal security amplifier was originally put forth to decided whether any efficient block cipher possessed a property of the one-time pad, namely that when composed with a non-perfect cipher was strictly more secure.

For security parameter $n$, let $\mathcal{M}^{\prime}=\left\{0, \ldots, 2^{n}\right\}$ and let $X$ and $Z$ independent universal security amplifiers on $\mathcal{M}=\left\{0, \ldots, 2^{n}-1\right\}$, so they leave fixed the final 
plaintext $2^{n}$. Let $Y$ deterministically pick out any permutation $\pi \notin \mathfrak{S}_{\mathcal{M}}$. For example $\pi$ could simply add $1 \bmod 2^{n}+1$, which is clearly computationally feasible. Now we'd like to compare $T=X Y Z$ with $D=X Z$. Since $X$ and $Z$ clearly have the group property, $\operatorname{supp}(d)=\mathfrak{S}_{\mathcal{M}}$, and so encrypting once the plaintext $2^{n}$ will yield ciphertext $2^{n}$ with $100 \%$ probability. The following is thus immediate.

Proposition 4 The product $D$ is distinguishable from any idealized cipher.

We may further exploit Thm. 2 and in this case, the expansion is huge.

Proposition 5 The alternating product cipher $T$ has $\operatorname{supp}(t)$ on about $\left(2^{n}+1\right)$ ! permutations.

Proof: Because the action $\mathfrak{S}_{\mathcal{M}^{\prime}}$ on $\mathcal{M}^{\prime}$ is multiply transitive we have by a standard result from group theory (see [15, Thm. 9.6]) that $\mathfrak{S}_{\mathcal{M}^{\prime}}=\mathfrak{S}_{\mathcal{M}} \cup \mathfrak{S}_{\mathcal{M}} \pi \mathfrak{S}_{\mathcal{M}}$, in other words the double coset relevant to Thm. 2, $\mathfrak{S}_{\mathcal{M}} \pi \mathfrak{S}_{\mathcal{M}}$ is nearly the whole of $\mathfrak{S}_{\mathcal{M}^{\prime}}$. This double coset has size $\left(2^{n}+1\right) !-2^{n} ! \approx\left(2^{n}+1\right) !$.

It remains then to show $t$ has full support on the double coset. But $z$ has full support on $\mathfrak{S}_{\mathcal{M}}$ [13], and by Thm. 2 each $z_{i} \preceq z$ so it cannot have fewer permutations on each of the left cosets $\lambda_{i} \mathfrak{S}_{\mathcal{M}}$. This forces $\operatorname{supp}(t)=\mathfrak{S}_{\mathcal{M}} \pi \mathfrak{S}_{\mathcal{M}}$, and we are done.

\section{References}

1. John Black. The ideal-cipher model, revisited: An uninstantiable blockcipher-based hash function. In Fast Software Encryption, pages 328-340. Springer, 2006.

2. Joseph Bonneau. Guessing human-chosen secrets. PhD thesis, University of Cambridge, May 2012.

3. Keith W. Campbell and Michael J. Wiener. DES is not a group. In Ernest F. Brickell, editor, Advances in Cryptology - CRYPTO '92, pages 512-517, Berlin, 1992. Springer-Verlag.

4. Ferdinando Cicalese and Ugo Vaccaro. Supermodularity and subadditivity properties of the entropy on the majorization lattice. Information Theory, IEEE Transactions on, 48(4):933-938, 2002.

5. Persi Diaconis. Group Representations in Probability and Statistics. Institute of Mathematical Statistics, Hayward, CA, 1988.

6. Peter Gaži and Ueli Maurer. Cascade encryption revisited. In Advances in Cryptology-ASIACRYPT 2009, pages 37-51. Springer, 2009.

7. B. Kaliski, R. Rivest, and A. Sherman. Is the Data Encryption Standard a group? (results of cycling experiments on DES). Journal of Cryptology, 1(1):1-36, 1988.

8. L.R. Knudsen and M.J.B. Robshaw. The block cipher companion. Springer-Verlag New York Inc, 2011.

9. Albert W. Marshall, Ingram Olkin, and Barry C Arnold. Inequalities: Theory of Majorization and Its Applications. Springer, New York, 2nd edition, 2011.

10. James L. Massey. Guessing and entropy. Proc. 1994 IEEE Int'l Symp. on Information Theory, page 204, 1994. 
11. Ben Morris, Phillip Rogaway, and Till Stegers. How to encipher messages on a small domain: Deterministic encryption and the Thorp shuffle. In Proceedings of the 29th Annual International Cryptology Conference on Advances in Cryptology - CRYTPO 2009, pages 286-302. Springer-Verlag, 2009.

12. John O. Pliam. On the Incomparability of Entropy and Marginal Guesswork in Brute-Force Attacks. In Proceedings of Indocrypt 2000, LNCS 1977, pages 67-79, Berlin, 2000. Springer-Verlag.

13. John O. Pliam. A Polynomial-Time Universal Security Amplifier in the Class of Block Ciphers. Communications in Information and Systems, 1(2):181-204, April 2001.

14. John O Pliam. Alternating Product Ciphers: A Case for Provable Security Comparisons. arXiv preprint arXiv:130\%.410\%, 2013. Full version of this paper.

15. Joseph J. Rotman. An Introduction to the Theory of Groups. Springer-Verlag, 4th edition, 1995.

16. Claude E. Shannon. Communication theory of secrecy systems. Bell System Tech. Jour., 28:656-715, 1949.

17. Serge Vaudenay. Decorrelation: a theory for block cipher security. Journal of Cryptology, 16(4):249-286, 2003.

18. Ralph Wernsdorf. The one-round functions of DES generate the alternating group. In R.A. Rueppel, editor, Advances in Cryptology - EUROCRYPT '92, pages 99112, Berlin, 1993. Springer-Verlag.

\section{Appendices}

\section{A.1 Variation Distance to Uniformity.}

The following lemma will prove quite useful.

Lemma 2 The variation distance to uniformity is Schur-convex.

Proof: Suppose $x \preceq y$ with $x, y \in \mathbb{R}_{+}^{n}$. As a consequence of the definition,

$$
\|u-x\|=\sum_{i=1}^{k_{x}} x_{[i]}-\frac{k_{x}}{n}, \text { and }\|u-y\|=\sum_{i=1}^{k_{y}} y_{[i]}-\frac{k_{y}}{n},
$$

where $k_{x}=\max \left\{i \mid x_{[i]} \geq 1 / n\right\}$ and $k_{y}=\max \left\{i \mid y_{[i]} \geq 1 / n\right\}$. If $k_{x}<k_{y}$, then

$$
\begin{aligned}
\sum_{i=1}^{k_{y}} y_{[i]}-\frac{k_{y}}{n} & =\sum_{i=1}^{k_{x}} y_{[i]}+\sum_{i=k_{x}+1}^{k_{y}} y_{[i]}-\left(\frac{k_{x}}{n}+\frac{k_{y}-k_{x}}{n}\right) \\
& =\sum_{i=1}^{k_{x}} y_{[i]}-\frac{k_{x}}{n}+\sum_{i=k_{x}+1}^{k_{y}}\left(y_{[i]}-\frac{1}{n}\right) \geq \sum_{i=1}^{k_{x}} x_{[i]}-\frac{k_{x}}{n} .
\end{aligned}
$$

I.e., $\|u-y\| \geq\|u-x\|$. In case $k_{x} \geq k_{y}$ the result follows mutatis mutandis. 


\section{A.2 Sketch of Proof of Thm. 1.}

Proof Sketch of Thm. 1: For arbitrary $q$-tuple $p \in \mathcal{M}^{(q)}$, let $H=\operatorname{Stab}_{G}(p)$, and let $\left\{g_{i}\right\}$ be a left transversal for $H$ in $G$. Then the two cases correspond to two different induced representations from $H$ to $G$. Specifically, $\mathbb{R} \uparrow_{H}^{G}$ describes distributions over $q$-tuples for comparing values of $\operatorname{Adv}_{X}^{\text {ncpa }}(q)$, while $\mathbb{R} H \uparrow_{H}^{G}$ describes the distributions over all permutations for comparing values of $W(X \mid C, p)$.

In either case, a general result adapted from [13, Lem. 3.3] is that if $z \preceq y$ in $V \uparrow_{H}^{G} \cong \bigoplus_{i} g_{i} \otimes V$ and the permutations for this majorization in (2) act on it by permuting direct summands $g_{i} \otimes V$ then

$$
z_{\downarrow}^{(i)}=\sum_{i=1}^{[G: H]} \omega_{i j} D_{i j} y_{\downarrow}^{(i)},
$$

where $z^{(i)}$ and $y^{(i)}$ are projections of $z$ and $y$ into the direct summands of $z$ and $y$, and where each $D_{i j}$ is doubly stochastic as is the matrix $\Omega=\left[\omega_{i j}\right]$.

(Case 1:) Define $\sigma$ taking $x \mapsto \hat{x}=\sum_{i} x_{\downarrow}^{(i)}$. The product relation assures (3), which by a result of Day [9, Prop. 5.A.6] implies $\hat{z} \preceq \hat{y}$. Since guesswork is Schur-concave and $W(X \mid C, p)=W\left(\sum_{i} x_{\downarrow}^{(i)}\right)$ we have $z \preceq_{q} y$.

(Case 2:) Define $\sigma$ taking $x \mapsto \hat{x}=\left[x\left(g_{1} H\right), \ldots, x\left(g_{[G: H]} H\right)\right]^{\mathrm{t}}$. Likely beginning with [17] and more recently [11] NCPA advantage $\mathbf{A d v}_{X}^{\text {ncpa }}(q)$ is identified with variation distance to uniformity $\|\hat{x}-\hat{u}\|$, which by Lem. 2 is Schur-convex. Now the action of $G$ on $G / H$ also gives rise to a left module action of $\mathbb{R} G$ on $\mathbb{R} \uparrow_{H}^{G}$ consistent with (3), now with 1 -dimensional summands. The $1 \times 1$ doubly stochastic matrices vanish and we obtain $\hat{z}=\Omega \hat{y}$ or $\hat{z} \preceq \hat{y}$. Again $z \preceq_{q} y$ by the Schur-convexity of $\mathbf{A d v}_{X}^{\text {ncpa }}(q)$. 